\title{
Spectroscopic investigations of the interaction of the anti-hypertension drug valsartan with human serum albumin
}

\author{
JIAN JING, XIN QU, ZHI TU, CHENHOO ZHENG and ZHICHENG ZHENG
}

Department of Bioorganic and Biological Chemistry, Beijing Key Lab, Beijing Normal University, Beijing 100875, P.R. China

Received August 16, 2013; Accepted March 4, 2014

DOI: $10.3892 / \mathrm{mmr} .2014 .2129$

\begin{abstract}
The aim of the present study was to investigate the interaction between valsartan, an anti-hypertension drug, and human serum albumin (HSA) using spectroscopic techniques, including fluorescence, ultraviolet-visible absorption, synchronous fluorescence and circular dichroism (CD). The results demonstrated that valsartan and HSA form a complex and that a static quenching mechanism occurs. In addition, the binding constant and the number of binding sites for valsartan on HSA were analyzed. Hydrophobic interactions and hydrogen bonds were the predominant forces in the association reaction based on thermodynamic parameters. The distance between the donor (HSA) and the acceptor (valsartan) was $1.994 \mathrm{~nm}$ as derived from Forster's theory. Alterations in the secondary structure of HSA in the presence of valsartan were assessed using synchronous fluorescence and CD. This study provides an enhanced understanding of the pharmacodynamic effects of valsartan on the physiologically important protein HSA.
\end{abstract}

\section{Introduction}

Serum albumins are the most prevalent soluble proteins found in vertebrates, and human serum albumin (HSA) is the predominant protein found in the plasma (1). HSA binds to a wide variety of substances, including metals, fatty acids, amino acids and hormones. The complexes formed between HSA and the ligands are involved in transport and regulatory processes (2). HSA consists of 585 amino acid residues and contains a single tryptophan $(\operatorname{Trp})$ residue, Trp-214, that invariably appears in the hydrophobic cavity of subdomain IIA (3).

Valsartan, which has the chemical formula $(S)-N$-oxopentyl- $N$ - $\{[2-(1 \mathrm{H}-5$-tetrazolyl)-4-di-phenyl]methyl $\}$-valine, is an orally potent specific non-peptide angiotensin II-receptor antagonist (Fig. 1). Valsartan has been shown to relax vascular

Correspondence to: Professor Jian Jing, Department of Bioorganic and Biological Chemistry, Beijing Key Lab, Beijing Normal University, 19 Xinwai Street, Beijing 100875, P.R. China

E-mail: jjing@bnu.edu.cn

Key words: valsartan, human serum albumin, interaction, spectroscopic investigations, binding characteristics smooth muscle, increase salt excretion, inhibit cell hypertrophy and lower blood pressure without changing the heart rate (4). As an anti-hypertension drug, the use of valsartan is effective against mild or moderate primary hypertension, particularly for kidney damage-induced secondary hypertension.

The interaction between a drug and plasma protein residues is capable of influencing the protein structure and function. Therefore, understanding the pharmacodynamics and pharmacokinetics of valsartan is of crucial importance. Investigations of this nature are likely to provide information about the structural features of valsartan that determine the therapeutic efficacy of the drug; such investigations may, therefore, become an important research field in chemistry, life sciences and clinical medicine. Several previous studies on fluorescence quenching and the mechanism of interaction between other drugs or bioactive small molecules and albumin have been reported (5-7). The knowledge gained from these studies has also been useful for the design of novel therapeutic agents (8). In the present study, the detailed mechanism underlying the interaction of valsartan with HSA was investigated using different spectroscopic techniques.

\section{Materials and methods}

Reagents and apparatus. HSA (fatty acid-free) was obtained from Sigma (St. Louis, MO, USA). The HSA solutions were prepared in $0.1 \mathrm{~mol} / 1$ phosphate buffer ( $\mathrm{pH}$ 7.4) containing $0.15 \mathrm{~mol} / 1 \mathrm{NaCl}$. HSA solutions were prepared based on the molecular weight of HSA (66,000 Da). Valsartan was obtained from Novartis (Copenhagen, Denmark). All other materials employed were of analytical grade, and Millipore water (EMD Millipore Corp., Billerica, MA, USA) was used throughout.

Fluorescence measurements were performed on a FluoroMax $^{\circledR}-4$ spectrofluorimeter (Horiba, Les Ulis, France) equipped with a $150 \mathrm{~W}$ xenon lamp and a slit width of $5 \mathrm{~nm}$. The circular dichroism (CD) measurements were made on a Jasco-J715 spectropolarimeter (Jasco, Tokyo, Japan) using a $0.1-\mathrm{cm}$ cell at $0.2-\mathrm{nm}$ intervals, with three scans for each CD spectrum between 200 and $260 \mathrm{~nm}$. The absorption spectra were recorded on a double-beam GBC Cintra-10e UV-Visible Spectrophotometer (Varian Medical Systems, Sydney, Australia) equipped with a $150 \mathrm{~W}$ xenon lamp and a slit width of $5 \mathrm{~nm}$.

Analysis of valsartan-HSA interaction. On the basis of preliminary experiments, fluorescence spectra were recorded between 
300 and $500 \mathrm{~nm}$, and 280 and $500 \mathrm{~nm}$ for HSA, respectively. The concentration of HSA was maintained at $1.0 \times 10^{-6} \mathrm{~mol} / 1$ and the concentration of valsartan varied between 1.0 and $10.0 \times 10^{-6} \mathrm{~mol} / 1$.

Ultraviolet-visible (UV-vis) absorption studies. The absorption spectra of HSA in the presence and absence of valsartan were recorded between 200 and $230 \mathrm{~nm}$. The concentration of HSA was maintained at $1.0 \times 10^{-6} \mathrm{~mol} / 1$ and the concentration of valsartan varied between 1.0 and $10.0 \times 10^{-6} \mathrm{~mol} / \mathrm{l}$.

Synchronous fluorescence measurements. The fluorescence studies were performed on a FluoroMax ${ }^{\circledR}-4$ spectrofluorimeter (Horiba). The spectra were recorded between 280 and $350 \mathrm{~nm}$. The synchronous fluorescence spectra were recorded with scanning ranges $\Delta \lambda=15 \mathrm{~nm}$ and $60 \mathrm{~nm}(\Delta \lambda=\lambda \mathrm{em}-\lambda \mathrm{ex}$, with $\lambda$ em representing the wavelength of the fluorescence emission spectra, and $\lambda$ ex referring to excitation wavelength) in the presence and absence of valsartan, to determine the spectrum characteristics of the protein residues.

Energy transfer between valsartan and HSA. The absorption spectrum of valsartan $\left(1.0 \times 10^{-6} \mathrm{~mol} / \mathrm{l}\right)$ was recorded between 300 and $550 \mathrm{~nm}$ and the emission spectrum of HSA $\left(1.0 \times 10^{-6} \mathrm{~mol} / \mathrm{l}\right)$ was recorded between 300 and $500 \mathrm{~nm}$. The overlap of the UV absorption spectrum of valsartan with the fluorescence emission spectrum of protein was then used to analyze the energy transfer.

Thermodynamics of valsartan-protein interactions. The thermodynamic parameters for the binding of valsartan to HSA were determined using binding studies at two different temperatures, 303 and $310 \mathrm{~K}$, in the range of 300-500 nm upon excitation at 296/280 $\mathrm{nm}$ using spectrofluorimetry.

\section{Results and Discussion}

Fluorescence quenching of HSA in the presence of valsartan. The interaction of valsartan with HSA ( $\mathrm{pH} 7.4)$ was analyzed by measuring the change in the intrinsic fluorescence intensity of HSA following the addition of valsartan (Fig. 2). A total of $10 \mathrm{ml}$ HSA working solution $(2.0 \mathrm{ml}$ buffer solution, $2.0 \mathrm{ml} \mathrm{NaCl}$ solution and $1.0 \mathrm{ml}$ HSA stock solution) was prepared. A total of $2.0 \mathrm{ml}$ working solution was placed in a $1-\mathrm{cm}$ quartz cell and a micro-injector was used to add the valsartan solution $\left(1.0 \times 10^{-4} \mathrm{~mol} / \mathrm{l}\right)$ gradually. No change in the fluorescence intensity was observed for the control HSA solution throughout the incubation period; however, as shown in Fig. 2, the fluorescence intensity of HSA at $340 \mathrm{~nm}$ decreased gradually as the concentration of valsartan increased. Fluorescence quenching refers to any process that decreases the fluorescence intensity of a sample (9) and occurs due to the interaction between HSA and valsartan. In the present study, a slight blue shift was observed for the emission wavelength as valsartan concentration increased. These results indicate that valsartan interacted with HSA and induce conformational changes in HSA. Different mechanisms of quenching are usually classified as either dynamic or static quenching, and they are distinguished by their differing dependence on temperature and viscosity (10).

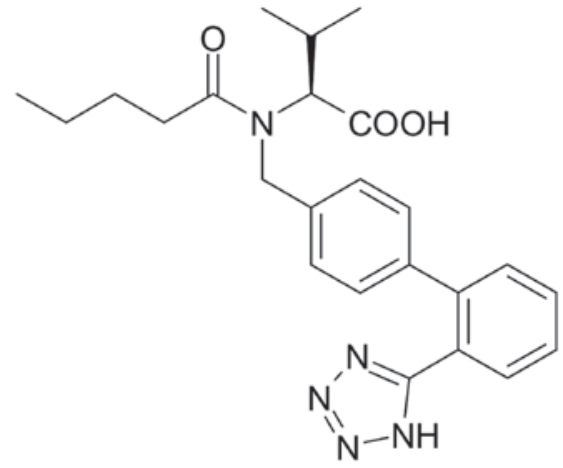

Figure 1. Structure of valsartan.

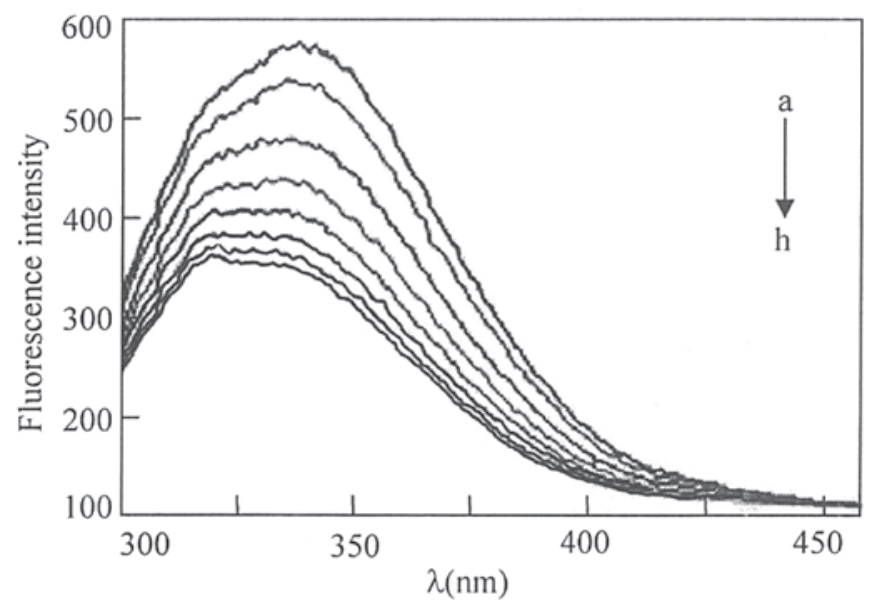

Figure 2. Fluorescence spectra of human serum albumin in the presence of valsartan. Concentration of HSA was fixed at $1.0 \times 10^{-6} \mathrm{~mol} / \mathrm{l}$ (a) and that of valsartan was maintained in the range of $1.0-10.0 \times 10^{-6} \mathrm{~mol} / \mathrm{l}(\mathrm{b}-\mathrm{h})$.

Dynamic quenching depends upon diffusion. Since higher temperatures result in larger diffusion coefficients, the bimolecular quenching constants are expected to increase with an increase in temperature. By contrast, increased temperature is likely to result in decreased stability of complexes, and thus lower values of quenching rate constants are observed for static quenching (11).

In order to investigate the fluorescence quenching mechanism, the fluorescence quenching data at different temperatures (303 and $310 \mathrm{~K}$ ) were firstly analyzed using the classical Stern-Volmer equation (12) as follows: $\mathrm{F}_{0} / \mathrm{F}=1+K_{s v}[$ valsartan $]=1+k_{q} \tau_{0}[$ valsartan $]$, where $\mathrm{F}_{0}$ and $F$ denote the fluorescence intensities in the absence and presence of valsartan, respectively, $K_{s v}$ is the Stern-Volmer quenching constant, $k_{q}$ is the quenching rate constant of HSA, $\tau_{0}$ is the average lifetime of the HSA without valsartan (the fluorescence lifetime of the biopolymer is $10^{-8} \mathrm{sec}$ ) (13), and [valsartan] is the concentration of valsartan. Fig. 3 shows the Stern-Volmer plots of $\mathrm{F}_{0} / \mathrm{F}$ versus [valsartan] at two temperatures, and the calculated $K_{s v}$ and $k_{q}$ values are presented in Table I. The results demonstrated that the Stern-Volmer quenching constant $K_{s v}$ was inversely correlated with the temperature, and that the values of $k_{q}$ were considerably larger than the maximum scattering collision quenching constant $\left(2.0 \times 10^{10} \mathrm{l} / \mathrm{mol} / \mathrm{sec}\right)(14)$. This indicated 
Table I. Stern-Volmer quenching constants for the interaction of valsartan with HSA.

\begin{tabular}{|c|c|c|c|c|}
\hline$T(\mathrm{~K})$ & $K_{s v}\left(\mathrm{x} 10^{4} 1 / \mathrm{mol}\right)$ & $k_{q}\left(\mathrm{x} 10^{12} 1 / \mathrm{mol} / \mathrm{sec}\right)$ & $R$ & SD \\
\hline 303 & 14 & 14 & 0.9973 & 0.010 \\
\hline 310 & 12 & 12 & 0.9841 & 0.009 \\
\hline
\end{tabular}

$T$, temperature; $K_{s v}$, Stern-Volmer quenching constant; $k_{q}$, quenching rate constant of HSA; $R$, correlation coefficient; SD, standard deviation; HSA, human serum albumin.

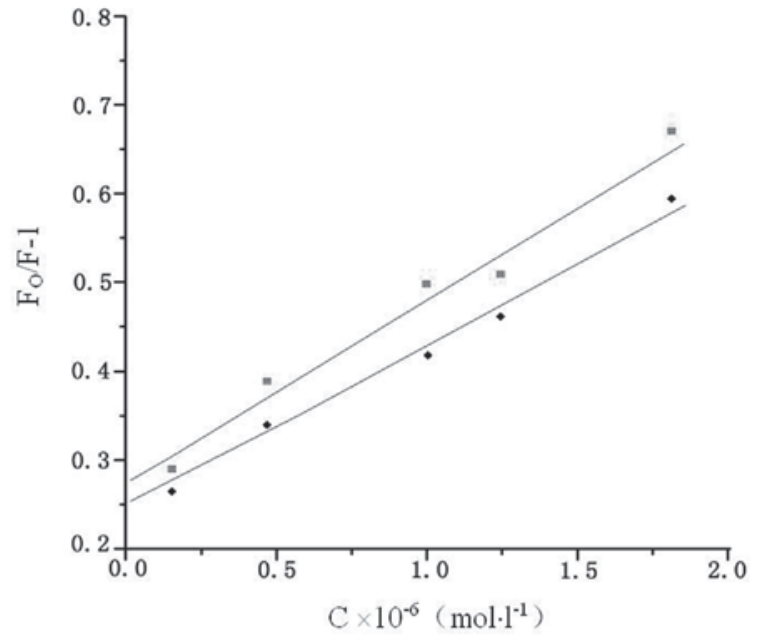

Figure 3. Stern-Volmer curves for quenching of HSA with valsartan at $303 \mathrm{~K}$ (indicated by the square) and $310 \mathrm{~K}$ (indicated by the rhombus). HSA, human serum albumin.

that the probable quenching mechanism of the fluorescence of HSA by valsartan was not initiated by dynamic collision but by compound formation (15).

Binding parameters. The binding constant $(K)$ and the number of binding sites $(n)$ were calculated using the following equation: $\log \left(F_{0}-F\right) / F=\log K+n \log [$ valsartan]. A plot of $\log \left[\left(F_{0}-F\right) / F\right]$ versus $\log [$ valsartan $]$ gives a straight line where the slope is equal to $n$ and the intercept on the $\mathrm{y}$ axis is equal to $\log K$ (Fig. 4). The values of $K$ and $n$ at 303 and $310 \mathrm{~K}$ are shown in Table II. The binding constant between valsartan and HSA decreased with increasing temperature, which suggests that an unstable complex exists between valsartan and HSA due to the static fluorescence quenching mechanism (16).

$U V$-Vis absorption. UV-Vis absorption spectra measurement is a simple method that is used to explore structural changes and complex formation (17). The absorbance of HSA increased with the addition of valsartan, whilst the absorption wavelength remained unchanged. This suggests that the interaction between valsartan and HSA led to a ground state complex formation and a change in the microenvironment around HSA.

Determination of the force acting between valsartan and HSA. Owing to the dependence of the binding constant on temperature, the thermodynamic parameters dependent on

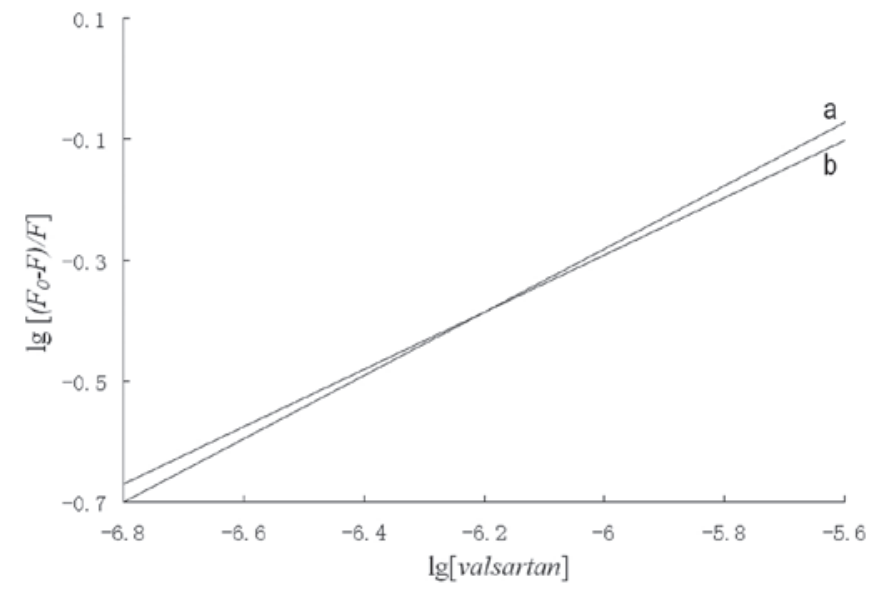

Figure 4. Plot of $\lg \left[\left(F_{0}-F\right) / F\right]$ versus $\lg [$ valsartan $]$ at (a) $303 \mathrm{~K}$ and (b) $310 \mathrm{~K}$.

temperature were analyzed in order to further characterize the acting force in the valsartan-HSA complex (18). The acting forces between small molecules and macromolecules primarily include hydrogen bonds, van der Waals forces, and electrostatic and hydrophobic interaction forces. The thermodynamic parameters of enthalpy change $(\Delta H)$, entropy change $(\Delta S)$ and free energy change $(\Delta G)$ are the main factors used to determine the binding mode (19). When the change in temperature is not clear, $\Delta \mathrm{H}$ is considered a constant. $\Delta \mathrm{H}$ and $\Delta \mathrm{S}$ values were calculated from the van't Hoff equation and obtained from a linear van't Hoff plot, as follows: $\ln K=-\Delta \mathrm{H} / R \mathrm{~T}+\Delta \mathrm{S} / R . K$ is analogous to the effective quenching constant $K_{s v}$ and $R$ is the gas constant. The free energy change $(\Delta G)$ was estimated from the following equation: $\Delta \mathrm{G}=\Delta \mathrm{H}-\mathrm{T} \Delta \mathrm{S}$. The results are shown in Table II. The positive $\Delta \mathrm{S}$ arose due to the more random arrangement of water molecules around HSA and valsartan, which was caused by hydrophobic interactions between valsartan and HSA. Negative $\Delta \mathrm{G}$ values revealed the spontaneity of the binding process. The negative $\mathrm{H}$ value $(-24.9 \mathrm{~kJ} / \mathrm{mol})$ was regarded as very small, almost zero, and so the interaction was not attributed to electrostatic forces (19). Therefore, from the thermodynamic characteristics summarized above, the negative $\Delta \mathrm{H}$ and positive $\Delta \mathrm{S}$ values indicate that hydrogen bonds and hydrophobic interaction have a major role in the valsartan-HSA binding reaction and contribute to the interaction and stability of the complex. The structures of serum albumins are particularly complex, so the interaction forces between valsartan and serum albumins may not be forces that are frequently observed; however, they may be more common under certain conditions. 
Table II. Binding constants and thermodynamic parameters of valsartan-human serum albumin interactions.

\begin{tabular}{ccccccc}
\hline$T(\mathrm{~K})$ & $K(\mathrm{l} / \mathrm{mol})$ & $n$ & $R^{2}$ & $\Delta H^{0}(\mathrm{~kJ} / \mathrm{mol})$ & $\Delta G^{0}(\mathrm{~kJ} / \mathrm{mol})$ & $\Delta S^{0}(\mathrm{~J} / \mathrm{mol} / \mathrm{K})$ \\
\hline 303 & 758.9 & 0.52 & 0.9877 & -24.9 & -35.9 & +36.3 \\
310 & 368.8 & 0.47 & 0.9938 & -24.9 & -36.2 & +36.3 \\
\hline
\end{tabular}

$T$, temperature; $K$, binding constant; $n$, number of binding sites; $R$, correlation coefficient; $\Delta H$, enthalpy change; $\Delta G$, free energy change; $\Delta S$, entropy change.

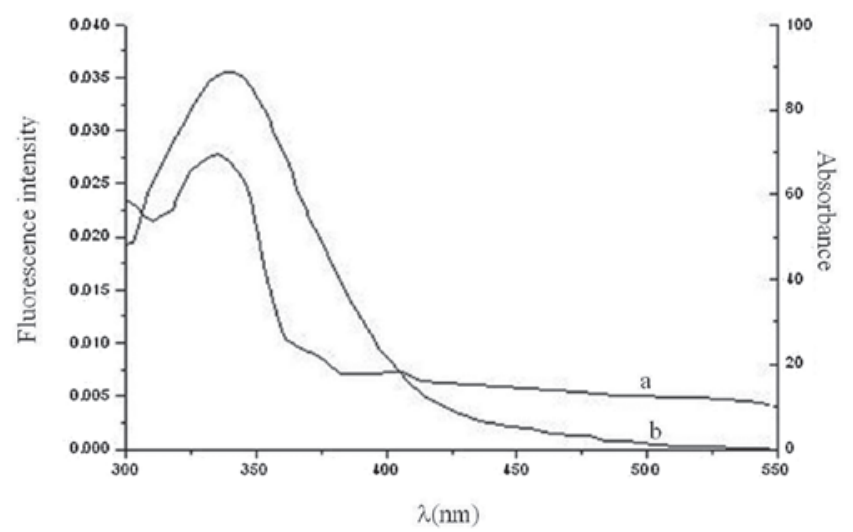

Figure 5. Overlap between the (a) ultraviolet absorption spectrum of valsartan and the (b) fluorescence spectrum of human serum albumin.

A

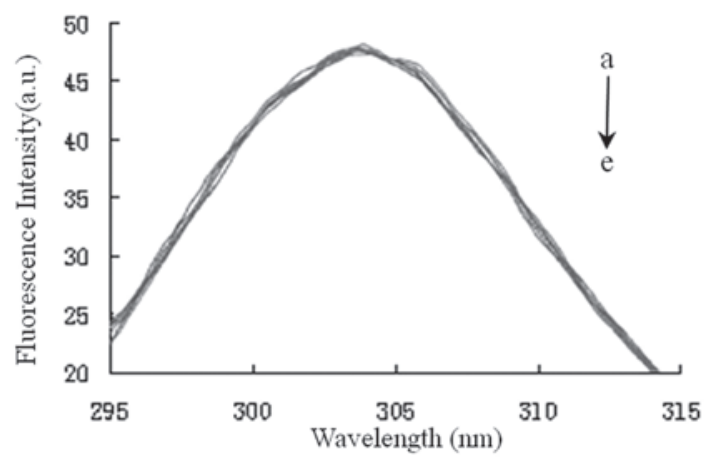

B

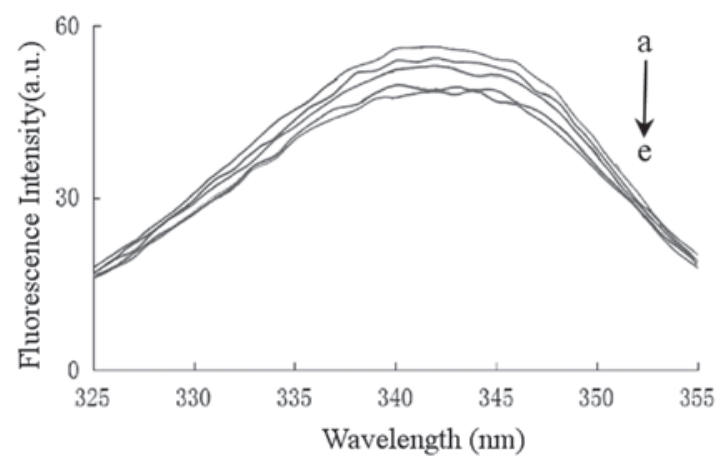

Figure 6. Synchronous fluorescence spectrometry of HSA as valsartan was added. a-e: $[$ valsartan $]=(1.0,1.5,2.0,3.0,5.0) \times 10^{-6} \mathrm{~mol} / 1$. $[\mathrm{HSA}]=1.0 \times 10^{-6} \mathrm{~mol} / 1$. (A) $\Delta \lambda=15 \mathrm{~nm}$; (B) $\Delta \lambda=60 \mathrm{~nm}$. HSA, human serum albumin.

Fluorescence resonance energy transfer (FRET). FRET is a nondestructive spectroscopic method that analyzes the proximity and relative angular orientation of fluorophores (20).

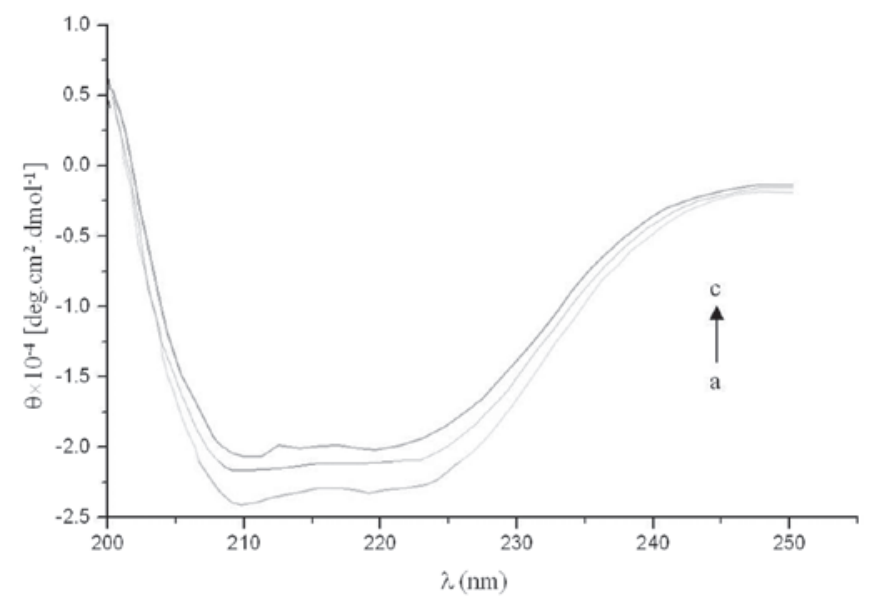

Figure 7. The far-ultraviolet circular dichroism spectra of the HSA-valsartan system. (a) HSA only $\left(1.0 \times 10^{-6} \mathrm{~mol} / \mathrm{l}\right)$, (b) HSA-valsartan $\left(3.0 \times 10^{-6} \mathrm{~mol} / \mathrm{l}\right)$ and (c) $\left(6.0 \times 10^{-6} \mathrm{~mol} / \mathrm{l}\right)$, respectively. HSA, human serum albumin.

According to the theory proposed by Förster (21), the efficiency of FRET depends mainly on the following factors: (i) The extent of overlap between the donor emission and the acceptor absorption spectrum; (ii) the orientation of the transition dipole of donor and acceptor and (iii) the distance between the donor and the acceptor. In the present study the donor and acceptor were HSA and valsartan, respectively. The overlap between the absorption spectrum of valsartan and the fluorescence emission spectrum of HSA is shown in Fig. 5.

The efficiency of energy transfer between the donor and acceptor, $E$, was calculated using the following equation: $E=1-F / F_{0}=R_{0}{ }^{6} /\left(R_{0}{ }^{6}+r^{6}\right)$, where $r$ is the binding distance between donor and acceptor and $R_{0}$ is the critical distance at $50 \%$ transfer efficiency. In addition, $R_{0}{ }^{6}$ may be calculated as follows: $R_{0}{ }^{6}=8.8 \times 10^{-25} K^{2} N^{-4} \phi J$, where $K^{2}$ is the orientation factor between the emission dipole of the donor and the absorption dipole of the acceptor, $N$ is the average refractive index of the medium in the wavelength range where the spectral overlap is significant, $\phi$ is the fluorescence quantum yield of the donor and $J$ is the overlap integral of the fluorescence emission spectrum of the donor and the absorption spectrum of the acceptor. Therefore, $J=\Sigma F(\lambda) \varepsilon(\lambda) \lambda^{4} \Delta \lambda / \Sigma F(\lambda) \Delta \lambda$, where $F(\lambda)$ is the fluorescence intensity of the donor in the wavelength range $\lambda$ to $\lambda+\Delta \lambda$, and $\varepsilon(\lambda)$ is the extinction coefficient of the acceptor at $\lambda$. In present study, $K^{2}=2 / 3, N=1.336$ and $\phi=0.12$ for HAS (22). From the equations, the values found were $J=2.02 \times 10^{-14} \mathrm{~cm}^{3} / 1 / \mathrm{mol}, R_{0}=1.792 \mathrm{~nm}, E=0.3410$ and $r=1.994 \mathrm{~nm}$. The donor-to-acceptor distance, $r$, was $<7 \mathrm{~nm}$ (23) and $0.5 R_{0}<r<1.5 R_{0}(24)$, which is in accordance with Förster's 
theory and suggests, with high probability, that nonradiative energy transfer from HSA to valsartan occurred (25). These results also indicate that valsartan strongly quenched the intrinsic fluorescence of HSA by the static quenching mechanism (26)

Conformational investigations by the synchronous fluorescence method. The synchronous fluorescence of HSA was investigated to evaluate the change in the microenvironments of tyrosine (Tyr) and Trp residues as a result of valsartan binding. The excitation and emission monochromators were synchronously scanned, separated by a constant wavelength interval $\Delta \lambda(\Delta \lambda=\lambda$ em- $\lambda$ ex $)$. When $\Delta \lambda=15 \mathrm{~nm}$, the spectrum characteristic of the protein Tyr residues was observed, and when $\Delta \lambda=60 \mathrm{~nm}$ the spectrum characteristic of protein Trp residues was observed (27). The fluorescence emission peaks for aromatic Tyr and Trp residues are sensitive to the polarity of their environment. Fig. 6 demonstrates the effect of the addition of valsartan on the synchronous fluorescence spectra of HSA when $\Delta \lambda=15$ or $60 \mathrm{~nm}$. Fig. 6 shows that the maximum emission wavelength of Tyr and Trp residues did not change significantly; however, the fluorescence intensity of Trp decreased, whereas that of Tyr remained almost unchanged. The results suggested that the binding between valsartan and the protein did not lead to a change in the polarity of the microenvironment of the Trp and Tyr residues; however, the internal packing of the protein changed (28). Furthermore, the results suggested that valsartan predominantly binds to Trp residues, but this binding does not appear to induce large structural changes in the microenvironment around the Trp residues.

$C D$ spectroscopy studies. The albumin structure is predominantly $\alpha$-helical. Approximately $60.78 \%$ of HSA is helical and the number of helices in the structure is 28 (29). CD spectra were used to analyze the interaction between valsartan and albumin. The results from the stepwise CD titration of albumin with increasing amounts of valsartan are shown in Fig. 7. The CD spectra of HSA exhibited two negative bands in the UV region at 209 and $222 \mathrm{~nm}$, characteristic of an $\alpha$-helical protein structure. The binding of valsartan to HSA distinctly decreased these bands. This indicated that considerable changes in the protein secondary structure had occurred, i.e. the decrease in the $\alpha$-helical content of the protein, which may have been the result of the formation of a complex between the protein and valsartan.

In conclusion, HSA is the main constituent of plasma proteins and is responsible for the binding and transport of numerous molecules, including valsartan. Drug binding to HSA is a major problem in pharmaceutical research since the binding to albumin influences the effective valsartan concentration that can obtained at the target site. In the present study the interaction of valsartan with HSA was investigated in vitro using fluorescence emission, UV-Vis, CD and synchronous fluorescence spectroscopy. The results from the fluorescence experiments revealed that the intrinsic fluorescence of HSA was quenched by a static quenching process. The changes in enthalpy $(\Delta \mathrm{H})$ and entropy $(\Delta \mathrm{S})$ for the reaction were found to be $-24.9 \mathrm{~kJ} / \mathrm{mol}$ and $36.3 \mathrm{~J} / \mathrm{mol} / \mathrm{K}$, suggesting that hydrophobic interactions and hydrogen bonds were the dominant inter-molecular forces stabilizing the complex. The distance between valsartan and HSA was sufficiently close $(r=1.994 \mathrm{~nm})$ to induce nonradiative energy transfer from HSA to valsartan. The results of the synchronous fluorescence and $C D$ spectra suggest that the protein secondary structure was altered and the physiological state of HSA was affected by valsartan. This binding study of valsartan with HSA is of biological importance and has particular significance for pharmacology and clinical medicine, as well as methodology.

\section{Acknowledgements}

This study was supported by the Fundamental Research Program of China Universities, Beijing Normal University (SD2010.1.1). The authors would like to thank Professor J.X. $\mathrm{Si}$, of the Academy of Medical Sciences, for her assistance during the $\mathrm{CD}$ measurement experiments.

\section{References}

1. Xiao J, Shi J, Cao H, Wu S, Ren F and Xu M: Analysis of binding interaction between puerarin and bovine serum albumin by multi-spectroscopic method. J Pharm Biomed Anal 45: 609-615, 2007.

2. He W, Li Y, Xue C, Hu Z, Chen X and Sheng F: Effect of Chinese medicine alpinetin on the structure of human serum albumin. Bioorg Med Chem 13: 1837-1845, 2005.

3. Sułkowska A, Maciazek M, Równicka J, Bojko B, Pentak D and Sułkowski WW: Effect of temperature on the methotrexate-BSA interaction: Spectroscopic study. J Mol Struct 834-836: 162-169, 2007.

4. Barakat K, Clark R and Davis M: Valsartan (eds): Hypertension and Cardiovascular Disease. CSF Medical Communications Ltd, Witney, 2004.

5. Mote US, Bhattar SL, Patil SR and Kolekar GB: Interaction between felodipine and bovine serum albumin: fluorescence quenching study. Luminescence 25: 1-8, 2010.

6. Varlan A and Hillebrand M: Bovine and human serum balbumin interactions with 3-carboxyphenoxathiin studied by fluorescence and circular dichroism spectroscopy. Molecules 15: 3905-3919, 2010.

7. Neelam S, Gokara M, Sudhamalla B, Amooru DG and Subramanym RJ: Interaction studies of coumaroyltyramine with human serum albumin and its biological importance. J Phys Chem B 114: 3005-3012, 2010.

8. He WY, Chen HJ, Sheng FL and Yao XJ: Molecular modeling and spectroscopic studies on binding of 2,6-bis[4-(4-amino-2trifluoromethylphenoxy)benzoyl] pyridine to human serum albumin. Spectrochim Acta A Mol Biomol Spectrosc 74: 427-433, 2009.

9. Silva D, Cortez CM, Cunha-Bastos J and Louro SR: Methyl parathion interaction with humanand bovine serum albumin. Toxicol Lett 147: 53-61, 2004.

10. Yue Y, Chen X, Qin J and Yao X: Spectroscopic investigation on the binding of antineoplastic drug oxaliplatin to human serum albumin and molecular modeling. Colloids Surf Biointerfaces 69: $51-57,2009$.

11. Lemma T and Pawliszyn J: Human serum albumin interaction with oxaliplatin studied by capillary isoelectric focusing with the whole column imaging detection and spectroscopic method. J Pharm Biomed Anal 50: 570-575, 2009.

12. Lakowicz JR (ed): Principles of Fluorescence Spectroscopy. 2nd edition. Kluwer Academic/ Plenum, New York, NY, 1999.

13. Lakowicz JR and Weber G: Quenching of fluorescence by oxygen. A probe for structural fluctuations in macromolecules. Biochemistry 12: 4161-4170, 1973.

14. Ware WR: Oxygen quenching of fluorescence in solution: An experimental study of the diffusion process. J Phys Chem 66: 455-458, 1962.

15. He Y, Wang Y, Tang L, Liu H, Chen W, Zheng Z and Zou G: Binding of puerarin to human serum albumin: a spectroscopic analysis and molecular docking. J Fluoresc 18: 433-442, 2008. 
16. Sarkar D, Mahata A, Das P, Girigoswami A, Ghosh D and Chattopadhyay N: Deciphering the perturbation of serum albumins by a ketocyanine dye: a spectroscopic approach. J Photochem Photobiol B 96: 136-143, 2009.

17. Wang YQ, Tang BP, Zhang HM, Zhou QH and Zhang GC: Studies on the interaction between imidacloprid and human serum albumin: spectroscopic approach. J Photochem Photobiol B 94: 183-190, 2009

18. Daneshgar P, Moosavi-Movahedi AA, Norouzi P, Ganjali MR, Madadkar-Sobhani A and Saboury AA: Molecular interaction of human serum albumin with paracetamol: spectroscopic and molecular modeling studies. Int J Biol Macromol 45: 129-134, 2009.

19. Ross PD and Subramanian S: Thermodynamics of protein association reactions: forces contributing to stability. Biochemistry 20: 3096-3102, 1981.

20. Stryer L and Haugland RP: Energy transfer: a spectroscopic ruler. Proc Natl Acad Sci USA 58: 719-726, 1967.

21. Förster T: Intermolecular energy migration and fluorescence. Ann Phys 437: 55-75, 1948 (In German).

22. Cyril L, Earl JK and Sperry WM (eds): Biochemists handbook. E \& FN Epon Led. Press, London, 1961.
23. Valeur B and Brochon JC (eds): New Trends in Influorescence Spectroscopy. 6th edition. Springer Press, Berlin, 1999.

24. Valeur B (ed): Molecular Fluorescence: Principles and Applications. Wiley Press, New York, NY, 2001.

25. Sun Y, Zhang H, Sun Y, Zhang Y, Liu H, Cheng J, Bi S and Zhang H: Study of interaction between protein and main active components in Citrus aurantium L. by optical spectroscopy. J Lumin 130: 270-279, 2010.

26. Abou-Zied OK and Alshihi OI: Characterization of subdomain IIA binding site of humanserum albumin in its native, unfolded, and refolded states using small molecular probes. J Am Chem Soc 130: 10793-10801, 2008.

27. Mandal P and Ganguly T: Fluorescence spectroscopic characterization of the interaction of human adult hemoglobin and two isatins, 1-methylisatin and 1-phenylisatin: a comparative study. J Phys Chem B 113: 14904-14913, 2009.

28. Grigoryan KR, Aznauryan MG, Bagramyan NA, Gevorkyan LG and Markaryan SA: Spectroscopic determination of binding between human serum albumin and a platinum(II) demethylsulfoxide complex. J Appl Spectrosc 75: 593-596, 2008.

29. Carter DC and Ho JX: Structure of serum albumin. Adv Protein Chem 45: 153-203, 1994. 